\title{
Psychosocial Assessment
}

National Cancer Institute

\section{Source}

National Cancer Institute. Psychosocial Assessment. NCI Thesaurus. Code C126879.

The act of determining, usually through interviews and observation, the state of an individual's psychological state and social environment. 